\section{Actividad física y perfil de estilos de vida promotores de la salud en adultos mayores chilenos}

\author{
SAMUEL DURÁN ${ }^{1, a, e}$, HUGO SÁNCHEZ $Z^{2, \mathrm{f}}$, \\ MACARENA VALLADARES ${ }^{3, b, e}$, ANTONIO LÓPEZ $Z^{4, c, e}$, \\ PABLO VALDÉS BADILLA ${ }^{5,6,7, \mathrm{~d}, \mathrm{~g},}$ TOMÁS HERRERA ${ }^{8,9, \mathrm{d,e}}$
}

\section{Association of sedentariness and health promoting lifestyles in older adults}

Background: Part of having healthy lifestyles should be a regular practice of physical activities. Aim: To evaluate the association between the practice of physical activity and healthy lifestyles in older people. Material and Methods: The Health-Promoting Lifestyle Profile survey was applied to 1688 older people living in the community, aged $72 \pm 7$ years (61\% women). This survey includes questions about regular physical activity. Results: Twenty four percent of surveyed participants declared to practice physical activities at least three times per week, without gender differences. Sedentariness increased along with age. There was a negative association between low physical activity and healthy lifestyles (odds ratio (OR): 3.2 confidence intervals (CI): 2.3-4.5). There was also an association between sedentariness and low self-realization (OR 2.4 CI: 1.8-3.3), low responsibility with health (OR 1.6 CI: 1.22.1), low concern about nutrition (OR 1.8 CI: 1.4-2.4), low stress management (OR 2.0 CI: 1.5-2.7) and low interpersonal support (OR 2.0 CI: 1.5-2.6). Conclusions: Sedentariness is negatively associated with health promoting lifestyles in this group of older people.

(Rev Med Chile 2017; 145: 1535-1540)

Key words: Aged; Exercise; Quality of Life.
${ }^{1}$ Facultad de Ciencias de la Salud. Universidad San Sebastián. Chile

${ }^{2}$ Instituto de Nutrición y Tecnología de los Alimentos (INTA), Universidad de Chile ${ }^{3}$ Centro Integrativo de Biología y Química Aplicada (CIBQA), Universidad Bernardo O Higgins, Chile. ${ }^{4}$ Kinesiólogo. Carrera de Kinesiología, Departamento Ciencias de la Salud, Facultad de Medicina, Pontificia Universidad Católica de Chile. Santiago, Chile.

IInstituto de Actividad Física y Salud, Universidad Autónoma de Chile. Chile.

${ }^{6}$ Pedagogía en Educación Física, Facultad de Educación, Universidad Autónoma de Chile, Temuco, Chile.

${ }^{7}$ Programa de Doctorado en Ciencias de la Actividad Física, Facultad de Ciencias de la Educación, Universidad Católica del Maule, Chile. ${ }^{8}$ Escuela de Ciencias de la Actividad Física, el Deporte y la Salud. Facultad de Ciencias Médicas. Universidad de Santiago de Chile, USACH. Chile.

${ }^{9}$ Escuela de Ciencias del Deporte. Facultad de Salud. Universidad Santo Tomás, UST. Chile.

a Nutricionista.

bBioquímica.

'Kinesiólogo.

'Profesor de Educación Física.

ePhD.

${ }^{\mathrm{f}} \mathrm{MPH}$.

${ }^{9} \mathrm{MSc}$.

Recibido el 5 de abril de 2017, aceptado el 29 de octubre de 2017.

Los autores no declaran conflictos de interés.

Trabajo no recibió financiamiento.

Correspondencia a

Samuel Durán Agüero

Universidad San Sebastián, Lota 4265,

Providencia, Chile.

samuel.duran@uss.cl

\section{L}

a población adulto mayor (AM) en Chile equivale a 1.171 .478 habitantes ${ }^{1}$, de los cuales $48,7 \%$ presenta una baja actividad física (AF) según la segunda Encuesta Nacional de Salud $^{2}$. La AF es recomendada como uno de los principales agentes favorecedores hacia un envejecimiento saludable, la que impactaría positivamente sobre la prevención y manejo de múltiples patologías asociadas con la vejez ${ }^{3}$.

$\mathrm{Al}$ respecto, existen pruebas convincentes que relacionan la práctica regular de AF de intensidad moderada a vigorosa con la reducción del riesgo de mortalidad por cualquier causa y la aparición de enfermedades crónicas como la demencia y depresión, además de contribuir con el bienestar social de los $\mathrm{AM}^{4,5}$. En directa relación con lo anterior, la $\mathrm{AF}$ en el AM se ha asociado con mejores indicadores que contribuyen a la disminución de factores de riesgo cardiometabólico ${ }^{6}$, cambios tanto en el estado nutricional (valorizado a través del menor índice de masa corporal-IMC-), como en la distribución de la grasa (mediante el uso de la circunferencia de cintura), al mismo tiempo produce modificaciones en la hemoglobina glicosilada y mayor HDL-colesterol ${ }^{7}$. Estos cambios en la salud están a menudo condicionados con 
la intensidad de la $\mathrm{AF}^{5}$, y asociados a un menor hábito tabáquico ${ }^{8}$.

Por otra parte, se ha considerado que la realización de al menos 30 minutos de AF de baja a moderada intensidad con una frecuencia de 5 o más días semanales, sería lo recomendado para lograr beneficios sobre la salud de las personas ${ }^{9,10}$; mientras que, el sedentarismo, condición que se caracteriza por un gasto energético bajo $(\leq 1,5$ MET equivalentes metabólicos), se ha asociado con efectos negativos sobre la salud ${ }^{11-14}$.

Diversas investigaciones han indagado sobre la calidad de vida en los $\mathrm{AM}^{15,16}$, sin embargo, $\mathrm{y}$ hasta donde conocemos, son pocos los trabajos relacionados con los estilos promotores de la salud. En este sentido, el objetivo del presente estudio pretende asociar la actividad física con los estilos de vida promotores de la salud en adultos mayores autónomos chilenos.

\section{Material y Métodos}

Estudio transversal. La muestra fue seleccionada por conveniencia y consistió en 1688 AM (60,6\% mujeres) de la Región Metropolitana (capital Santiago) de Chile, quienes fueron reclutados en Centros de Salud Familiar pertenecientes a las comunas de: Recoleta, Macul, Santiago Centro, La Florida, Puente Alto, Providencia, Pedro Aguirre Cerda, La Granja, Cerro Navia, La Reina, Pudahuel, Melipilla, Quilicura, Conchalí, San Bernardo, La Cisterna y Buin.

Se incluyeron en el estudio AM de ambos sexos, con 60 o más años de edad y autónomos. Se diagnosticó como autónomos a los AM que obtuvieron $\geq 43$ puntos de acuerdo a la prueba de funcionalidad EFAM-Chile ${ }^{17}$, quienes además presentaban la capacidad para comprender y seguir instrucciones. Todos los AM firmaron un consentimiento informado que autorizaba el uso de la información con fines científicos. Los procedimientos de evaluación y tratamiento de datos fueron previamente revisados y aprobados por el Comité de Ética de la Universidad San Sebastián de Chile (Proyecto 2013-0007-c).

\section{Encuesta}

A los participantes se les aplicó la encuesta de Estilo de Vida y Promotor de Salud (EVPS), instrumento validado en español ${ }^{18}$. La encuesta evalúa las dimensiones de autorrealización, res- ponsabilidad en salud, ejercicio, nutrición, apoyo interpersonal y manejo del estrés. Ésta consiste en 48 aseveraciones sobre estilo de vida o hábitos personales, con 4 posibilidades de respuesta en escala de tipo Likert (nunca, a veces, frecuentemente y siempre). El puntaje total de esta encuesta oscilaba entre 48 y 192 puntos, a mayor puntuación, mejor EVPS ostenta el AM evaluado.

\section{Evaluación del estado nutricional}

Se efectuó una evaluación antropométrica de peso corporal y estatura bípeda. La determinación del peso se realizó con un mínimo de ropa, utilizando una balanza electrónica de precisión (GAM$\mathrm{MA}^{\circledR}$ ), capacidad máxima de $220 \mathrm{~kg}$, precisión en $50 \mathrm{~g}$. La estatura se midió con un tallímetro que está incorporado a la balanza (precisión $0,1 \mathrm{~cm}$ ). Se calculó el IMC para establecer la clasificación ponderal del estado nutricional en AM de acuerdo a lo recomendado por la Organización Mundial de la Salud ${ }^{19}$, de esta manera, se distribuyó a los participantes en: bajo peso/normopeso $(\leq 24,9$ $\left.\mathrm{kg} / \mathrm{m}^{2}\right)$; sobrepeso $\left(25 \mathrm{a} 29,9 \mathrm{~kg} / \mathrm{m}^{2}\right)$; y obesidad $\left(\geq 30 \mathrm{~kg} / \mathrm{m}^{2}\right)$.

\section{Estadística}

Para las variables continuas se realizó la prueba de Shapiro Wilk para determinar la normalidad de las variables. En la encuesta EVPS y en las subdimensiones se consideró mal estilo promotor de salud cuando el puntaje era $\leq$ al percentil 25 (criterio de los investigadores, la encuesta no entrega un punto de corte), de esta manera se obtuvo valores dicotómicos de buen estilo de vida promotor de la salud $=0 \mathrm{y}$ mal estilo de vida promotor de la salud $=1$. Para evaluar la asociación existente entre AF con cada una de las dimensiones de los estilos de vida, se realizó una regresión logística, la cual fue ajustada por edad $(>0<75$ años), sexo (hombre/mujer), estado nutricional (normal/sobrepeso-obeso), consumo de tabaco (consume/no consume) y cantidad de sueño ( $>0<7 \mathrm{~h})$. Para el análisis estadístico se utilizó el programa SPSS versión 22.0, considerando significativo un valor de $\mathrm{p}<0,05$.

\section{Resultados}

Del total de AM encuestados $(\mathrm{n}=1.688)$ se observaron valores promedios para la edad de 71,5 \pm 7,4 años, peso corporal de $69,5 \pm 11,8 \mathrm{~kg}$, estatura 
bípeda de 1,60 $\pm 0,08 \mathrm{~m}$ e IMC de 26,8 $\pm 4,2 \mathrm{~kg} / \mathrm{m}^{2}$ (Tabla 1). El 23,9\% de los AM declara realizar AF al menos 3 veces por semana. Se observa además que, el porcentaje de sujetos activos era similar en ambos sexos (cercano a 24\%), el consumo de tabaco fue de $17,6 \%$ en mujeres y $23,4 \%$ en hombres. Datos que pueden ser apreciados en la Tabla 2.

$\mathrm{Al}$ comparar los AM activos frente a los sedentarios se observa en la Figura 1 que, el grupo de mayor edad presenta más sedentarismo $(80 \%)$ respecto a los más jóvenes $(74 \%)(p=0,003)$, los
AM activos consumen menos tabaco que los sedentarios $25 \%$ versus $20 \%$ ( $\mathrm{p}=0,038$ ), la ingesta de alcohol entre los sujetos activos es más baja en relación a los sedentarios. Por otra parte, se advierte una disminución en el porcentaje de sujetos activos a medida que aumenta el IMC (para este análisis fueron eliminados los sujetos clasificados con bajo peso), reportando $27 \%$ de normopeso, $23 \%$ de sobrepeso y $20 \%$ de obesidad $(\mathrm{p}=0,055)$, sin lograr significancia estadística.

En la Tabla 3, se observa que, la baja AF se

Tabla 1. Caracterización antropométrica y estilos de vida promotores de la salud en adultos mayores autónomos chilenos

\begin{tabular}{|lcccc|}
\hline & \multicolumn{2}{c}{ Mujeres } & \multicolumn{2}{c|}{ Hombres } \\
& $\begin{array}{c}\text { Activas } \\
(\mathbf{n = 2 4 3 )}\end{array}$ & $\begin{array}{c}\text { Sedentarias } \\
(\mathbf{n = 7 6 8 )}\end{array}$ & $\begin{array}{c}\text { Activos } \\
(\mathbf{n = 1 6 2})\end{array}$ & $\begin{array}{c}\text { Sedentarios } \\
(\mathbf{n = 5 1 5 )}\end{array}$ \\
\hline Edad (años) & $69,2 \pm 6,4$ & $71,7 \pm 7,8$ & $71,6 \pm 6,5$ & $72,1 \pm 7,5$ \\
\hline Peso corporal $(\mathrm{kg})$ & $64,1 \pm 9,8$ & $66,9 \pm 11.6$ & $73,1 \pm 8,7$ & $74,8 \pm 11,5$ \\
\hline Estatura bípeda (m) & $1,55 \pm 0,06$ & $1,56 \pm 0,07$ & $1,68 \pm 0,07$ & $1,67 \pm 0,07$ \\
\hline IMC (kg/m ${ }^{2}$ ) & $26,5 \pm 4,3$ & $27,1 \pm 4,6$ & $25,8 \pm 2,9$ & $26,7 \pm 3,7$ \\
\hline Autorrealización & $37,8 \pm 7,8$ & $39,0 \pm 7,9$ & $37,2 \pm 7,9$ & $39,2 \pm 7,3$ \\
\hline Responsabilidad en salud & $24,4 \pm 6,7$ & $23,9 \pm 6,1$ & $23,9 \pm 6,3$ & $23,5 \pm 6.1$ \\
\hline Ejercicio & $10,1 \pm 4,0$ & $9,5 \pm 3,8$ & $9,9 \pm 3,8$ & $9,6 \pm 3,9$ \\
\hline Nutrición & $16,9 \pm 3,4$ & $16,8 \pm 3,2$ & $17,2 \pm 3,7$ & $17,0 \pm 3,1$ \\
\hline Apoyo interpersonal & $20,2 \pm 4,2$ & $20,4 \pm 3,9$ & $19,8 \pm 4,0$ & $20,6 \pm 4,0$ \\
\hline Estrés & $17,8 \pm 4,6$ & $17,7 \pm 4,1$ & $18,2 \pm 4,5$ & $17,7 \pm 4,0$ \\
\hline Calidad de Vida & $127,6 \pm 23,2$ & $127,7 \pm 21,5$ & $126,7 \pm 22,7$ & $128,2 \pm 20,3$ \\
\hline
\end{tabular}

Valores expresados en media $\pm \mathrm{DE}$.

Tabla 2. Clasificación del estado nutricional y hábitos de vida en adultos mayores autónomos chilenos

\begin{tabular}{|c|c|c|c|c|}
\hline & \multicolumn{2}{|c|}{ Mujeres } & \multicolumn{2}{|c|}{ Hombres } \\
\hline & $\begin{array}{c}\text { Activas } \\
\mathbf{n}\end{array}$ & $\begin{array}{c}\text { Sedentarias } \\
\%\end{array}$ & $\begin{array}{c}\text { Activos } \\
\mathbf{n}\end{array}$ & $\begin{array}{c}\text { Sedentarios } \\
\%\end{array}$ \\
\hline \multicolumn{5}{|l|}{ Estado nutricional } \\
\hline Bajo peso & 5 & 0,4 & 2 & 0,2 \\
\hline Normal & 367 & 36,3 & 235 & 34,7 \\
\hline Sobrepeso & 417 & 41,2 & 329 & 48,5 \\
\hline Obesidad & 222 & 21,9 & 111 & 16,3 \\
\hline Consumo de tabaco (Sí) & 178 & 17,6 & 159 & 23,4 \\
\hline Sueño (> 7 h) & 270 & 26,7 & 189 & 27,9 \\
\hline Consumo alcohol (Sí) & 38 & 3,7 & 67 & 9,8 \\
\hline Actividad física (si) & 243 & 24,3 & 162 & 23,9 \\
\hline
\end{tabular}


(a) 100

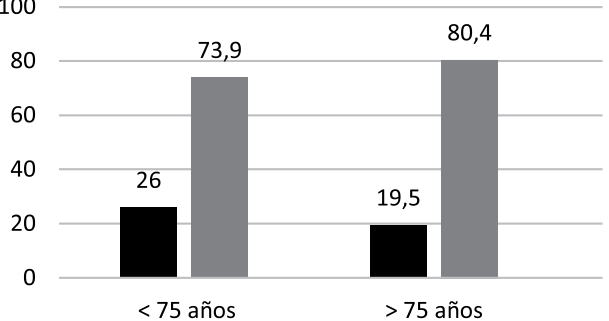

(c) 100

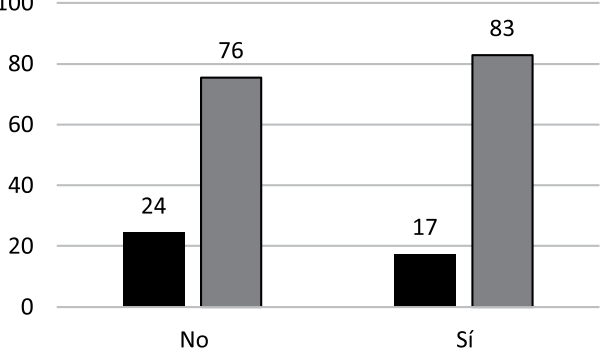

(b)

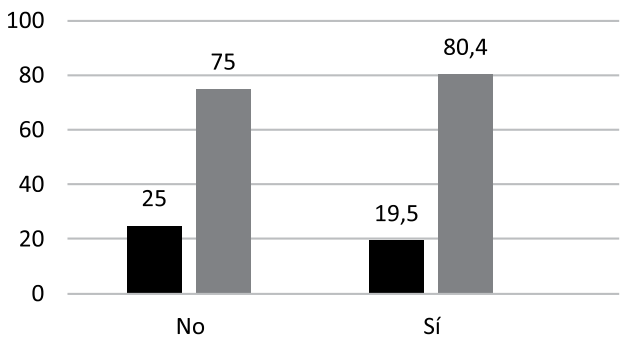

Sedentario

(d) 100

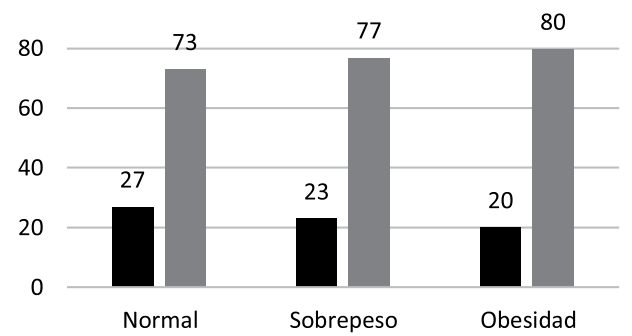

Figura 1. Comparación entre adultos mayores chilenos activos y sedentarios. a) Comparación entre \% sujetos $>0<$ de 75 años según actividad física $\left(\chi^{2}=0,003\right)$; b) Comparación entre \% consumo de tabaco según actividad física $\left(\chi^{2}=0,038\right)$; c) Comparación $\%$ de sujetos que consumen alcohol según actividad física $\left(\chi^{2}=0,054\right)$ y d) Comparación \% sujetos según estado nutricional y actividad física $\left(\chi^{2}=0,055\right)$.

Tabla 3. Asociación entre baja actividad física con las dimensiones de los estilos de vida promotores de la salud en adultos mayores autónomos chilenos

\begin{tabular}{|lcc|}
\hline \multicolumn{2}{|l}{$\begin{array}{c}\text { Baja actividad física } \\
\text { OR }\end{array}$} & IC $95 \%$ \\
\hline Baja calidad de vida & 3,24 & $2,31-4,52$ \\
Dimensiones & & \\
Baja autorrealización & 2,44 & $1,81-3,31$ \\
Responsabilidad & 1,62 & $1,23-2,13$ \\
Baja preocupación nutrición & 1,84 & $1,42-2,39$ \\
Bajo manejo del estrés & 2,0 & $1,53-2,67$ \\
Bajo apoyo interpersonal & 2,0 & $1,52-2,62$ \\
\hline
\end{tabular}

Ajustado por sexo, estado nutricional, consumo de tabaco y sueño. asocia negativamente con los EVPS y con las subdimensiones de baja autorrealización, responsabilidad en salud, baja preocupación con nutrición, bajo manejo del estrés y bajo apoyo interpersonal.

\section{Discusión}

El principal resultado del estudio señala que, una baja $\mathrm{AF}$ se relaciona negativamente con los estilos de vida promotores de la salud en AM autónomos chilenos.

En concordancia con nuestros resultados, un estudio previo realizado en AM indonesios también mostró que la $\mathrm{AF}$ al igual que la edad y el sexo, están asociados a los estilos de vida promotores de la salud ${ }^{20}$.

La AF regular puede ser considerada como uno de los elementos más importantes para la salud de 
las personas, especialmente, en los $\mathrm{AM}^{21}$. De esta manera, la AF favorece la reducción de factores de riesgo generales ${ }^{22}$ y de mortalidad ${ }^{23}$. En este sentido, nuestros resultados son coincidentes con trabajos previos ${ }^{24,25}$, dado a que los AM que ostentan mayor AF, poseen también, más probabilidades de presentar un mejor estilo de vida. Por lo tanto, la salud pública debería generar acciones para incentivar la práctica de $\mathrm{AF}$ en los $\mathrm{AM}$ como una herramienta de promoción de la salud ${ }^{22}$, debido a proteger y mejorar la salud física y mental de los individuos ${ }^{26}$. Además, reduce los síntomas de depresión, mejora la satisfacción con la vida y promueve una mejor calidad de vida ${ }^{27}$. Hecho coincidente con los resultados de los AM activos evaluados en nuestra investigación. Otro estudio que incluyó AM activos mostró en comparación con el grupo físicamente inactivo, que el OR para multimorbididad era de 0,84 (IC 95\% 0,78-0,91) en $\mathrm{AF}$ leve, $\mathrm{OR}=0,61$ (IC 95\% 0,56-a 0,66) con AF moderada y OR=0,45 (IC 95\% 0,41-0,49) en la $\mathrm{AF}$ vigorosa ${ }^{28}$.

Del mismo modo, una investigación con 85 AM los cuales fueron clasificados según su nivel de AF, reportó que los sujetos con AF moderada e intensa, además de presentar un mejor perfil lipídico, mostraban niveles séricos más elevados de vitamina $\mathrm{D}$, calcio y capacidad total antioxidante ${ }^{29}$. La mayoría de los estudios informaron la correlación positiva entre vitamina $\mathrm{D}$ y el rendimiento muscular, tales como la resistencia a la fuerza muscular y la función en los ancianos ${ }^{30}$. Otro estudio, de seguimiento en AM, reportó que realizar AF moderada a vigorosa tenía una probabilidad significativamente menor de incidencia de padecer sarcopenia $(\mathrm{OR}=0,64$, IC 95\% 0,45-0,91), además los AM con AF moderada a vigorosa tenían mayores niveles basales de masa muscular, fuerza y una marcha de mayor velocidad ${ }^{31}$. Situación auspiciosa para los AM activos de nuestra investigación.

Según la Encuesta Nacional de Hábitos de Actividad Física y Deportes ${ }^{32}$, la práctica de actividad física de las personas entre 60 y 69 años es de $16,9 \%$, la que disminuye a $16,5 \%$ en el grupo mayor de 70 años. Esta tendencia decrece a medida que aumentan los años, lo cual significa que con el tiempo se van haciendo relevantes otras razones, como los problemas de salud o la enfermedad ${ }^{32}$.

Entre las fortalezas del estudio podemos señalar que, se trabajó con un alto número de sujetos con características similares, junto con utilizar una encuesta validada internacionalmente. Mientras que, la principal limitación sería el diseño del estudio, que solo permite hablar de asociación, pero no de causalidad.

\section{Conclusión}

El sedentarismo se asocia negativamente con todos los aspectos referentes a los estilos de vida promotores de la salud en AM autónomos chilenos, hecho que sugiere fomentar la práctica regular de AF, no solo como un componente de prevención, sino que también, como un elemento de intervención social, lo que podría favorecer las redes de apoyo y la calidad de vida de los AM.

\section{Referencias}

1. Instituto Nacional de Estadísticas de Chile (INE) disponible en: http://www.ine.cl/canales/chile_estadistico/ estadisticas_sociales_culturales/adultos mayores/adultos_mayores.php.

2. II Encuesta Nacional de salud. Chile http://www. minsal.gob.cl/portal/url/item/bcb03d7bc28b64dfe040010165012d23.pdf

3. World Health Organization. Global recommendations on physical activity for health. Switzerland: World Health Organization; 2010.

4. Yorston LC, Kolt GS, Rosenkranz RR. Physical activity and physical functioning older adults: the 45 and Up Study. J Am Geriatr Soc 2012; 60: 719-25.

5. World Health Organization. Good health adds life to years: Global brief for World Health Day 2012. http:// www.who.int/ageing/publications/whd2012_global_ brief/en/2012.

6. Bakrania K, Edwardson CL, Bodicoat DH, Esliger DW, Gill JM, Kazi A, et al. Associations of mutually exclusive categories of physical activity and sedentary time with markers of cardiometabolic health in English adults: a cross-sectional analysis of the Health Survey for England. BMC Public Health 2016; 16: 25.

7. Santana Jde O, Ramalho JR, Firmo JO, Lima-Costa MF, Peixoto SV. Physical activity and Framingham risk score in older adults: the Bambui Health and Aging Study. Cad Saude Publica 2015; 31 (10): 2235-40.

8. Zaitune MPA, Barros MBA, Lima MG, César CLG, Carandina L, Goldbaum M, et al. Fatores associados ao tabagismo em idosos: inquérito de saúde no Estado de São Paulo (ISA-SP). Cad Saude Publica 2012; 28: 58396. 
9. Buman MP, Hekler EB, Haskell WL, Pruitt L, Conway $\mathrm{TL}$, Cain KL, et al. Objective light-intensity physical activity associations with rated health in older adults. Am J Epidemiol 2010; 172: 1155-65.

10. Department of Health, UK. Physical activity, health improvement and prevention: at least five a week. London, UK: Department of Health, 2004.

11. Biswas A, Oh PI, Faulkner GE, Bajaj RR, Silver MA, Mitchell MS, et al. Sedentary time and its association with risk for disease incidence, mortality, and hospitalization in adults: a systematic review and meta-analysis. Ann Intern Med 2015;162 (2): 123-32.

12. Thorp AA, Owen N, Neuhaus M, Dunstan DW. Sedentary behaviors and subsequent health outcomes in adults a systematic review of longitudinal studies, 1996-2011. Am J Prev Med 2011; 41 (2): 207-15.

13. Wilmot EG, Edwardson CL, Achana FA, Davies MJ, Gorely T, Gray LJ, et al. Sedentary time in adults and the association with diabetes, cardiovascular disease and death: systematic review and meta-analysis. Diabetologia 2012; 55 (11): 2895-905.

14. Zhai L, Zhang Y, Zhang D. Sedentary behaviour and the risk of depression: a meta-analysis. Br J Sports Med 2015; 49 (11): 705-9.

15. Roehr S, Luck T, Pabst A, Bickel H, König HH, et al. Subjective cognitive decline is longitudinally associated with lower health-related quality of life. Int Psychogeriatr 20171-12.

16. Pan CW, Cong X, Zhou HJ, Li J, Sun HP, Xu Y, Wang P. Self-Reported Sleep Quality, Duration, and Health-Related Quality of Life in Older Chinese: Evidence From a Rural Town in Suzhou, China. J Clin Sleep Med 2017. pii: jc-17-00057. [Epub ahead of print]

17. Manual de Aplicación del Examen de Medicina Preventiva del Adulto Mayor. (Chile) disponible en: http://www.minsal.cl/portal/url/item/ab1f81f43ef0c2a6e04001011e011907.pdf.

18. Walker SN, Kerr MJ, Pender NJ, Sechrist KR.A Spanish language version of the Health-Promoting Lifestyle Profile. Nurs Res 1990; 39 (5): 268-73.

19. Organization. WH. Obesity: preventing and managing the global epidemic. Report of a WHO Consultation. Geneva: World Health Organization; 2000

20. Onunkwor OF, Al-Dubai SA, George PP, Arokiasamy J, Yadav H, Barua A, et al. A cross-sectional study on quality of life among the elderly in non-governmental organizations' elderly homes in Kuala Lumpur. Health Qual Life Outcomes 2016; 14: 6.

21. Bayati M. Physical Inactivity and Sedentary Lifestyle. Iranian Journal of Endocrinology and Metabolism. 2012; 13 (5): 537-9.
22. Sørensen M, Gill DL. Perceived barriers to physical activity across Norwegian adult age groups, gender and stages of change. Scand J Med Sci Sports 2008; 18 (5): 651-63.

23. Young Ho K, Bradley J C. Effects of a transtheoretical model-based stage-matched intervention to promote physical activity among Korean adults. Int J Clin Health Psychol 2009; 9 (2): 259-73.

24. Schuch FB, Vancampfort D, Rosenbaum S, Richards J, Ward PB, Veronese N, et al. Exercise for depression in older adults: a meta-analysis of randomized controlled trials adjusting for publication bias. Rev Bras Psiquiatr. 2016; 38 (3): 247-54.

25. Ludyga S, Gerber M, Brand S, Holsboer-Trachsler E, Pühse U. Acute effects of moderate aerobic exercise on specific aspects of executive function in different age and fitness groups: A meta-analysis. Psychophysiology. 2016; 53 (11): 1611-1626.

26. Azizan A, Justine M, Siew Kuan Ch. Effects of a Behavioral Program on exercise Adherence and exercise Self-Efficacy in Community-Dwelling Older Persons. Curr Gerontol Geriatr Res 2013; 1-10.

27. Haraldstad K, Rohde G, Stea TH, Lohne-Seiler H, Hetlelid K, Paulsen G, Berntsen S. Changes in health-related quality of life in elderly men after 12 weeks of strength training. Eur Rev Aging Phys Act 2017; 14: 8.

28. Dhalwani NN, O’Donovan G, Zaccardi F, Hamer M, Yates T, Davies M, et al. Long terms trends of multimorbidity and association with physical activity in older English population. Int J Behav Nutr Phys Act 2016; 13: 8.

29. Al-Eisa ES, Alghadir AH, Gabr SA. Correlation between vitamin D levels and muscle fatigue risk factors based on physical activity in healthy older adults. Clin Interv Aging 2016; 11: 513-22.

30. Verreijen AM, Verlaan S, Engberink MF, Swinkels S, de Vogel-van den Bosch J, et al. A high whey protein-, leucine-, and vitamin D-enriched supplement preserves muscle mass during intentional weight loss in obese older adults: a double-blind randomized controlled trial. Am J Clin Nutr 2015; 101 (2): 279-86.

31. Mijnarends DM, Koster A, Schols JM, Meijers JM, Halfens RJ, Gudnason V, et al. Physical activity and incidence of sarcopenia: the population-based AGES-Reykjavik Study. Age Ageing 2016; 45 (5): 614-20.

32. Ministerio del deporte. Encuesta Nacional de Hábitos de Actividad Física y Deportes 2015 [documento en internet]. Santiago: MINDEP, 2016 [consultado en octubre de 2016]. Disponible en: http://www.mindep. cl/wp-content/uploads/2016/07/PRESENTACION-ENCUESTA-HABITOS-2015.pdf. 